\title{
CARACTERÍSTICAS DO PROCESSO DE TRABALHO DO ENFERMEIRO EM PRONTO-ATENDIMENTO
}

\author{
Mitzy Tannia Reichembach Danski ${ }^{1}$, Larissa Walter de Oliveira ${ }^{2}$, Priscila Mingorance ${ }^{3}$, Edivane Pedrolo ${ }^{4}$, Luciana
} Souza Marques de Lazzari ${ }^{5}$, Derdried Athanasio Johann ${ }^{6}$

\begin{abstract}
RESUMO: Estudo descritivo cujo objetivo foi identificar as atividades realizadas pelo enfermeiro assistencial em uma Unidade de Pronto Atendimento de um hospital universitário de Curitiba/PR. A coleta de dados foi realizada por observação não participante, durante seis horas diárias, pelo período de um mês. Foram elencadas as atividades rotineiras do enfermeiro com vistas a caracterizar o seu processo de trabalho. O enfermeiro dispõe de $61 \%$ de seu tempo para a realização de atividades gerenciais, $21 \%$ para as atividades assistenciais, $12 \%$ de cuidado pessoal e $6 \%$ no ensino e pesquisa. Mesmo desenvolvendo atividades assistenciais, a função gerencial é intrínseca ao trabalho do profissional enfermeiro, caracterizando-o como referência para a equipe no que diz respeito à gerência da unidade. Assim, percebe-se que o planejamento das atividades é essencial para o desenvolvimento do processo de trabalho do enfermeiro.

PALAVRAS-CHAVE: Enfermeiras; Prática profissional; Processos de enfermagem.

\section{CHARACTERISTICS OF THE NURSE'S WORK PROCESS IN ACCIDENT AND EMERGENCY}

\begin{abstract}
A descriptive study whose aim was to identify the activities carried out by Accident and Emergency nurses in an Accident and Emergency Unit in a university hospital in Curitiba in the state of Paraná. Data collection was by nonparticipant observation for six hours a day for the period of one month. The nurses' routine activities were classified with a view to characterising their work processes. Nurses spend $61 \%$ of their time on management activities, $21 \%$ on helping others, $12 \%$ on personal care, and $6 \%$ on teaching and research. Even considering the time spent on helping others, the management role is intrinsic to the work of nursing professionals, characterizing them as a reference to the team in terms of the unit's management. In this way it may be seen that the planning of activities is essential to the development of the nurse's work process.
\end{abstract}

KEYWORDS: Nurses; Professional practice; Nursing processes.

\section{CARACTERÍSTICAS DEL PROCESO DE TRABAJO DEL ENFERMERO EN HOSPITAL DE URGENCIAS}

RESUMEN: Estudio descriptivo cuyo objetivo fue identificar las actividades realizadas por el enfermero asistencial en una Unidad de Hospital de Urgencias de un hospital universitario de Curitiba/PR. Los datos fueron recogidos por medio de observación no participante, durante seis horas al día, por el periodo de un mes. Fueron apuntadas las actividades rutineras del enfermero con fines de caracterizar su proceso de trabajo. El enfermero dispone de $61 \%$ de su tiempo para la realización de actividades administrativas, $21 \%$ para las actividades asistenciales, $12 \%$ de cuidado personal y $6 \%$ en la enseñanza e investigación. Mismo desarrollando actividades asistenciales, la función administrativa está intrínseca al trabajo del profesional enfermero, caracterizándose como referencia para el equipo acerca de la administración de la unidad. Así, se puede percibir que el planeamiento de las actividades es esencial para el desarrollo del proceso de trabajo del enfermero. PALABRAS CLAVE: Enfermeros; Práctica profesional; Procesos de enfermería.

\footnotetext{
${ }^{1}$ Enfermeira. Doutora em História. Professora deo Departamento de Enfermagem e do Programa de Pós-Graduação em Enfermagem da Universidade Federal do Paraná-PPGENF UFPR. Vice-líder do Grupo de Pesquisa Tecnologia e Inovação em Saúde: Fundamentos para a Prática Profissional-TIS UPFR.

${ }^{2}$ Enfermeira.

${ }^{3}$ Acadêmica de Enfermagem. Bolsista de Iniciação Científica TN UFPR. Membro do TIS UFPR.

${ }^{4}$ Enfermeira. Professora do Instituto Federal do Paraná. Membro do TIS UFPR.

${ }^{5}$ Acadêmica de Enfermagem. Aluna Voluntária de Iniciação Científica. Membro do TIS UFPR.

${ }^{6}$ Enfermeira do Instituto Federal do Paraná. Mestranda no PPGENF UFPR. Membro do TIS UFPR.
}

\author{
Autor correspondente: \\ Priscila Mingorance \\ Universidade Federal do Paraná \\ R. Padre Camargo, 120 - 80060-240 - Curitiba-PR-Brasil \\ E-mail: primingo@yahoo.com.br
}

Recebido: $24 / 04 / 10$

Aprovado: 03/02/11 


\section{INTRODUÇÃO}

A Enfermagem é um campo de conhecimento voltado para busca, manutenção e restauração da saúde dos indivíduos no âmbito familiar, hospitalar e comunitário, tendo como pressuposto o "cuidar em enfermagem". Baseia suas atividades na prevenção e promoção da saúde, na recuperação da doença e na reabilitação do doente e de sua família, desde o seu nascimento até a morte.

Desenvolve ações técnicas, com bases científicas, para identificação e intervenção em situações clínicas específicas; coordenação e administração do cuidado interdisciplinar e do ambiente terapêutico; investigação epidemiológica, sociológica, administrativa, demográfica e de ensino ${ }^{(1)}$.

O enfermeiro é o profissional responsável pela avaliação, identificação e intervenção de enfermagem em indivíduos, pautadas por conhecimentos científicos próprios. Ele utiliza a liderança, o trabalho em equipe e os conhecimentos científicos para gerenciar, coordenar e intervir no cuidado nas diferentes fases da vida e do processo saúde-doença, devendo ter domínio intelectual da dinâmica da unidade e, inclusive, do contexto da prática ${ }^{(2)}$.

A prática da Enfermagem moderna surgiu na segunda metade do século XIX, com a figura de Florence Nightingale, e foi dividida em dois segmentos: quem realizava o cuidado propriamente dito e quem supervisionava essas atividades, sendo o ensino uma ação das supervisoras. Atualmente, considera-se que o processo de trabalho de enfermagem está dividido em quatro subprocessos: cuidar ou assistir, administrar ou gerenciar, pesquisar e ensinar ${ }^{(3)}$. Esses campos são interligados na prática profissional, possuem um corpo científico próprio que os fundamenta e possibilita o desenvolvimento de um cuidado de qualidade ao paciente.

Os diversos papeis exercidos pelo enfermeiro acarretam, em suas interfaces, responsabilidades específicas. Entretanto, todas essas ações são interligadas e sobrepostas, sendo o cuidado ao ser humano o foco do trabalho assistencial. O processo de trabalho do enfermeiro está intimamente ligado à atuação profissional, pois incorpora elementos específicos da prática (objetos; instrumentos; finalidades; e produtos) (4-5), variáveis de acordo com o campo de atuação.

Este profissional é constantemente influenciado em sua prática e no processo de trabalho pelo contexto em que está inserido, tanto econômico, político e social, quanto cultural. Cita-se o contexto organizacional como exemplo de fator que influencia e limita o desempenho das atribuições do enfermeiro, por vezes restringindo sua atuação a um dos subprocessos de trabalho: gerenciar ou cuidar ${ }^{(6)}$.

Considera-se o processo de trabalho como a maneira pela qual o homem constroi e retrata sua existência ${ }^{(7)}$. É por meio deste processo que as relações sociais são estabelecidas para o indivíduo que vive em sociedade. Os elementos componentes do processo de trabalho são: o trabalho (atividade com fim específico); o objeto de trabalho (matéria necessária para o seu desenvolvimento); e o meio de trabalho (instrumentos imprescindíveis ao trabalho) ${ }^{(8)}$.

Para a pesquisa, utilizou-se as seguintes definições: objeto é tudo "aquilo sobre o que se trabalha"; é o produto em potencial, ou seja, é a base da transformação efetuada pelo ser humano com o trabalho. Instrumentos são meios que o indivíduo emprega para modificar a natureza; eles podem ser tangíveis, como uma seringa, um estetoscópio, ou intangíveis, como o conhecimento científico, o saber da Enfermagem ou o processo de enfermagem. Finalidade do trabalho é o objetivo da realização do trabalho, aquilo que dá significado e é a razão de sua existência. Por fim, Produtos do Trabalho podem ser bens materiais ou serviços prestados aos indivíduos ${ }^{(5: 222)}$. Nesta pesquisa, entende-se a atuação profissional do enfermeiro dentro de um fluxo de trabalho que varia conforme as necessidades de cada Instituição.

Considera-se que o enfermeiro assistencial é aquele cujo objeto de trabalho é o cuidado exercido a pessoas e suas famílias, grupos e coletividades nas dimensões física, psicológica, social e espiritual, utilizando seus instrumentos próprios ${ }^{(5)}$. O processo de enfermagem é um dos instrumentos utilizados no processo de trabalho do enfermeiro para embasar e direcionar as ações de cuidado direto ao paciente ${ }^{(9)}$. As ações do enfermeiro assistencial contemplam, ainda, a supervisão dos membros de diferentes níveis de formação que compõem a equipe de enfermagem, e também a realização de procedimentos invasivos e complexos. As intervenções desempenhadas pelo enfermeiro assistencial visam atingir a finalidade do subprocesso assistir/cuidar, a melhora do estado de saúde do paciente.

É evidente que o enfermeiro assistencial, além de realizar cuidados diretos aos pacientes, organiza os cuidados de assistência indireta; assim, para concretizar suas funções, utiliza, também, instrumentos 
gerenciais e administrativos que estruturam, planejam, organizam e viabilizam a execução do cuidado.

Entende-se que a Enfermagem administrativa ou gerencial pode ser dividida em quatro dimensões: técnica, na qual o enfermeiro planeja, coordena, supervisiona, controla e avalia tanto os recursos humanos como os materiais e físicos; política, em que articulam-se determinantes internos de trabalho e externos da sociedade, de caráter político-ideológicos, econômicos, organizações corporativas e diversidade de usuários dos serviços, para o bom desenvolvimento do setor; comunicativa, na qual se estabelece uma relação de trabalho entre a equipe de saúde e a comunidade; e a dimensão de desenvolvimento da cidadania. Além das atividades citadas, o enfermeiro administrativo tem como objetivo instituir o cuidado ao paciente, organizar o ambiente e os profissionais técnicos em enfermagem, treinando-os e utilizando as técnicas disciplinares ${ }^{(3)}$.

Além disso, é preciso considerar a importância da pesquisa e do ensino, pois por meio deles o enfermeiro produz e transmite conhecimentos técnicos e científicos atualizados que colaboram e embasam a prática de enfermagem ${ }^{(3-10)}$. No processo de trabalho em enfermagem, a pesquisa figura como peça-chave para o desenvolvimento da ciência Enfermagem, ao produzir ou aprimorar tecnologias para o cuidado, para a gerência e para o ensino.

Neste contexto, a presente pesquisa teve por objetivos descrever o processo de trabalho do enfermeiro assistencial de um Serviço de Pronto-Atendimento e identificar as atividades realizadas pelo enfermeiro assistencial em seu processo de trabalho, categorizandoas.

\section{METODOLOGIA}

Trata-se de uma pesquisa de natureza descritiva, desenvolvida no Serviço de Pronto-Atendimento de um hospital universitário de Curitiba/PR, que possui um profissional alocado como "enfermeiro assistencial" em cada turno de trabalho. O sujeito de pesquisa foi o enfermeiro assistencial do turno vespertino. Vale considerar que as atividades gerenciais são realizadas pelo enfermeiro administrativo. Para esta pesquisa, entende-se o termo "enfermeiro" como "enfermeiro assistencial" (sujeito da pesquisa).

A coleta de dados ocorreu por meio da observação sistemática não participante diária, com duração de 6 horas (13:00h às 19:00h), de segunda a sexta-feira, pelo período de um mês (07/08 a 05/09/2007), totalizando 23 dias. O registro das informações foi realizado por meio de um instrumento criado pelos autores e que contempla a descrição das atividades desempenhadas e o tempo dispendido em cada uma delas.

Os dados coletados foram agrupados em categorias $^{(4)}$, desmembrados de acordo com os componentes do processo de trabalho da enfermagem e analisados, comparando-se o preconizado pela instituição e o realizado pelo enfermeiro.

A pesquisa foi aprovada pelo Comitê de Ética em Pesquisa com seres Humanos de uma Universidade Federal, como parte da primeira etapa do Projeto de Iniciação Científica "Avaliação das Práticas do Cuidado da Enfermagem" a que está vinculada, sob o número CAAE 0048.0.091.000-07. Considerou-se, para desenvolvimento da pesquisa, a Resolução n. 196/96 do Conselho Nacional de Saúde.

\section{RESULTADOS}

A unidade escolhida para a coleta de dados possui em seu quadro de funcionários de enfermagem dois cargos específicos para os profissionais de nível superior: enfermeiros assistenciais e enfermeiros administrativos, que possuem institucionalmente delimitadas as funções que exercem. Esta divisão de atividades auxiliou a observação, pois, possibilitou o foco do trabalho no profissional alocado como enfermeiro assistencial.

As atividades observadas foram categorizadas de acordo com os subprocessos descritos como sendo práticas do trabalho do enfermeiro ${ }^{(8)}$ : atividades de gerência; assistência; de ensino e pesquisa; e cuidado pessoal.

Atividades de Gerência: esta categoria englobou duas subcategorias: "Gerenciamento do Cuidado" e "Gerenciamento da Unidade". Ambas subcategorias incluem atividades de gerência e exigem conhecimento específico nesta área. Categorizaram-se na subcategoria "Gerenciamento do Cuidado" as seguintes ações: contato com a equipe de enfermagem - supervisão de procedimentos, dúvidas, orientações relacionadas a pacientes/cuidados - escalas de atividades, organização da ficha do paciente, recebimento e passagem de plantão, procedimentos relativos a óbito, higienização das mãos, preparo de material e auxílio em procedimento. Organizados na subcategoria "Gerenciamento da Unidade" estão as ações: contato com outros profissionais, escala de trabalho, ligações telefônicas, 
agendamentos e transferências, controle de materiais e equipamentos, organização do ambiente, controles administrativos e envolvimento com a equipe.

Atividades de Assistência: nesta categoria, incluíram-se todas as atividades relacionadas ao cuidado direto ao paciente. As atividades elencadas foram: avaliação do paciente, entrevista sem registro, monitorização de paciente, realização do processo de enfermagem, visitas aos pacientes e realização de cuidados em geral - sondagem nasoenteral, acesso venoso periférico, sondagem vesical de demora, entre outros. No entanto, se visualizou a realização incompleta do processo de enfermagem, esta que é uma tecnologia instrumental do processo de trabalho do enfermeiro.

Atividades de Ensino e Pesquisa: as ações observadas e categorizadas neste subprocesso de trabalho do enfermeiro foram: atividades educativas como orientações à equipe, pacientes e familiares/ acompanhantes, treinamentos, orientações e supervisão a acadêmicos de enfermagem, participação em pesquisas científicas e aperfeiçoamento profissional por meio de cursos específicos na área de atuação como, por exemplo, a participação em congressos.

Atividades de Cuidado Pessoal: esta categoria diz respeito a todas as atividades relacionadas ao bem-estar do profissional. Evidenciou-se, neste trabalho, que estas atividades constituem parte significativa da atuação do enfermeiro, devido à sua frequência semanal e mensal. Dentre as atividades observadas incluem-se: toalete, alimentação, conversas informais, ligações telefônicas pessoais, acesso à internet e saídas antecipadas do horário de trabalho para fins pessoais.

Abaixo, apresentamos a porcentagem do tempo semanal dispensado em cada categoria, bem como o tempo total (Quadro 1).

Em relação às subcategorias das Atividades

Quadro 1 - Porcentagem de tempo destinado a cada categoria do processo de trabalho de enfermeiros. Curitiba, 2007

\begin{tabular}{lcccccc}
\hline Atividades & Semana 1 & Semana 2 & Semana 3 & Semana 4 & Semana 5 & Total da Pesquisa \\
\hline Gerência & $67 \%$ & $66 \%$ & $66 \%$ & $58 \%$ & $49 \%$ & $61 \%$ \\
Assistência & $24 \%$ & $21 \%$ & $26 \%$ & $16 \%$ & $16 \%$ & $21 \%$ \\
Ensino e Pesquisa & $3 \%$ & $3 \%$ & $1 \%$ & $2 \%$ & $26 \%$ & $6 \%$ \\
Cuidado Pessoal & $6 \%$ & $10 \%$ & $7 \%$ & $24 \%$ & $9 \%$ & $12 \%$ \\
\hline
\end{tabular}

Gerenciais (Gerência da Unidade e Gerência do Cuidado), o gráfico 1 demonstra a relação entre ambas, considerando-se o período total de observação.

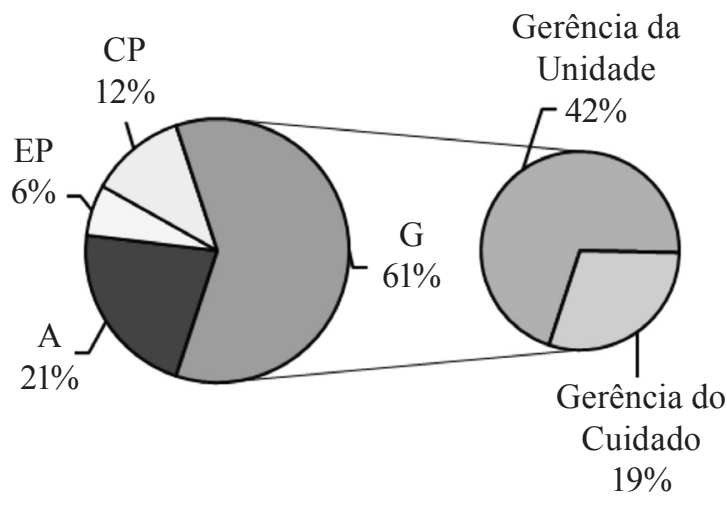

Gráfico 1 - Porcentagem de tempo destinado à atividades de Gerência da Unidade e Gerência do Cuidado. Curitiba, 2007

\section{DISCUSSÃO}

Discutiu-se os resultados agrupados por categoria, relacionou-se os dados obtidos aos possíveis fatores de interferência.

Ao observar o tempo mensal gasto na realização das atividades, aponta-se que o enfermeiro realiza, em grande parte de seu tempo, atividades categorizadas como atividades de gerência. As três primeiras semanas de observação revelaram que mais de $60 \%$ do tempo do turno de trabalho do enfermeiro foi despendido nessas atividades. Ao final da pesquisa, observou-se que as atividades de Gerência consumiram $61 \%$ de todo o tempo do enfermeiro, o que pontua mais da metade das atividades realizadas por este profissional.

Fica evidente que o tempo destinado às atividades de Gerência da Unidade é superior ao tempo destinado às atividades de Gerência do Cuidado.

As ações categorizadas como Atividades de Assistência são, depois das Gerenciais, as mais realizadas pelo profissional, tendo consumido $21 \%$ do tempo 
total. Embora as atividades assistenciais ocupem a segunda maior porcentagem de tempo do enfermeiro, percebeu-se que a quarta e a quinta semana foram atípicas. Durante estas semanas, o enfermeiro necessitou sair mais cedo de seu turno de trabalho para realização de atividades pessoais e também ausentar-se do trabalho para participação em Congresso relacionado à área de atuação. Estes fatos aumentaram as percentagens em suas respectivas categorias (Atividades de Cuidado Pessoal e Atividades de Ensino e Pesquisa).

As ações pertencentes à categoria de Atividades de Cuidado Pessoal ocuparam 12\% do tempo total, o que caracteriza a terceira categoria mais realizada pelo enfermeiro. Pode-se, assim, visualizar que o descanso durante a jornada de trabalho, preconizado pela Consolidação das Leis Trabalhistas $\left(\right.$ CLT) ${ }^{(11)}$, deve ser incluído no processo de trabalho do enfermeiro, pois demanda tempo do trabalhador.

As ações menos realizadas pelo enfermeiro durante esse período de observação foram aquelas relacionadas à categoria de Atividades de Ensino e Pesquisa, correspondendo a $6 \%$ do tempo total observado. Esta categoria é a que menos aparece durante todo o período de observação, exceto na última semana, conforme já mencionado.

\section{CONCLUSÃo}

Os dados revelam que o enfermeiro destinado às atividades assistenciais despende grande parte de seu tempo em atividades gerenciais, sendo a Gerência da Unidade a principal delas. Este fato pode estar relacionado à ausência de um enfermeiro administrativo no período vespertino.

Um aspecto que pode ser observado é que a equipe de enfermagem tem o profissional enfermeiro como referência no que diz respeito à gerência da unidade, de modo que este deve saber se comunicar, ter espírito de liderança, motivar, conhecer e ter entrosamento com a equipe que trabalha; mas, principalmente, ele deve planejar as atividades do turno a fim de que possa atender as demandas diárias.

Outro fator associado ao aumento do tempo despendido nas Atividades de Gerência e, consequentemente, à diminuição das Atividades de Assistência, é o fato de que o Serviço de Pronto-Atendimento em questão está estruturado fisicamente em dois andares e, durante o turno da tarde, está sob a supervisão de um único enfermeiro assistencial, que desempenha atividades de assistência e também de gerência. Este profissional é o responsável por todos os pacientes, pelo controle de materiais, equipamentos, notificação de intercorrências, problemas estruturais e funcionários nestes dois ambientes da unidade.

$\mathrm{O}$ ensino de Enfermagem enfatiza a necessidade da realização do Processo de Enfermagem, incluindo suas etapas: avaliação do paciente e coleta de dados; estabelecimento de diagnósticos de enfermagem; escolha de intervenções de enfermagem; e avaliação dos resultados. Compreende-se que é por meio do Processo de Enfermagem que o enfermeiro conquista autonomia e visibilidade profissional. A observação permitiu a constatação de que estes conhecimentos não são aplicados na realidade observada e que as demandas urgentes sobrecarregam o enfermeiro, fazendo com que ele se perca em seu processo de trabalho. Com base nos dados obtidos, identificou-se que o enfermeiro contratado como assistencial não despende a maior parte de seu tempo na dimensão prática do cuidar, mas sim no gerenciar, devido às demandas da unidade.

A ausência do enfermeiro na execução da assistência de enfermagem contribui para o desconhecimento da população quanto ao papel do enfermeiro assistencial. Defende-se que o enfermeiro é o profissional que possui a competência técnica e conhecimento científico para prestar os cuidados de enfermagem necessários e dar assistência a pacientes graves, e que tal assistência será de melhor qualidade quando executada por este profissional.

O Processo de Enfermagem é uma tecnologia privativa do enfermeiro, e que define a profissão como ciência. $\mathrm{O}$ abandono desse processo descaracteriza o cuidado de enfermagem, impede a Enfermagem de ocupar seu papel social e promove o desempenho de uma prática submissa e desqualificada.

Há que se repensar o processo de trabalho em enfermagem na dimensão prática do cuidar, pois, como observado nesta pesquisa, muitas vezes a falta de planejamento do processo de trabalho sobrecarrega o enfermeiro com demandas e assuntos urgentes, fazendo com que este dispense grande parte do seu tempo de trabalho para resolução de problemas relacionados à prática de enfermagem.

Ademais, observou-se o não-cumprimento das normas institucionais, considerando-se que o sujeito da pesquisa não desempenha as funções pré-estabelecidas para seu cargo, fator decorrente da ausência do enfermeiro administrativo para o bom andamento do serviço. 


\section{REFERÊNCIAS}

1. Lima MJ. O que é enfermagem. $3^{\mathrm{a}}$ ed. São Paulo: Brasiliense; 2005.

2. Domenico EBL, Ide CAC. Projetos de formandos em enfermagem: representações do vir a ser profissional. In: Ide CAC, Domenico EBL. Ensinando e aprendendo um novo estilo de cuidar. São Paulo: Atheneu; 2001. p. 4.

3. Felli VEA, Peduzzi M. O trabalho gerencial em enfermagem. In: Kurcgant P, coordenadora. Gerenciamento em enfermagem. Rio de Janeiro: Guanabara Koogan; 2005. p. 1-13.

4. Argenta IM, Pires DEP. Refletindo o processo de trabalho de enfermagem em um hospital público. Texto \& Contexto Enferm. 2000;9(2):288-97.

5. Sanna MC. Os processos de trabalho em enfermagem. Rev Bras Enferm. 2007;60(2):221-4.

6. Shimbo AY, Lacerda MR, Labronici LM. Processo de trabalho do enfermeiro em unidade de internação hospitalar: desafios de uma administração contemporânea. Cogitare Enferm. 2008;13(2):296-300.

7. Horta WA. Processo de enfermagem. São Paulo: EPU; 1979.

8. Marx K. O capital. Trad. de J. Teixeira Martins e Vital Moreira. Coimbra: Centelha; 1974.

9. Brandalize DL; Kalinowski CE. Processo de enfermagem: vivência na implantação da fase de diagnóstico. Cogitare Enferm. 2005;10(3):53-7.

10. Saar SRC, Trevizan MA. Papéis profissionais de uma equipe de saúde: visão de seus integrantes. Rev LatinoAm Enfermagem. 2007;15(1):106-12.

11. Brasil. Decreto n. 5.452 , de $1^{\circ}$ de maio de 1943 , aprova a consolidação das leis do trabalho. Diário Oficial da União [Internet] 09 ago 1943 [acesso em 03 abr 2008]. Disponível: http://www.planalto.gov.br/ccivil/decretolei/del5452.htm 